\section{Mortalidade por doenças cerebrovasculares por residência e local de ocorrência do óbito: Paraná, Brasil, 2007}

\author{
Stroke mortality by residence and place of death: \\ Paraná State, Brazil, 2007
}

\author{
1 Faculdade de Saúde \\ Pública, Universidade de São \\ Paulo, São Paulo, Brasil. \\ 2 Departamento de \\ Enfermagem, Universidade \\ Estadual de Maringá, \\ Maringá, Brasil. \\ Correspondência \\ T. S. Furukawa \\ Faculdade de Saúde Pública, \\ Universidade de São Paulo. \\ Av. Dr. Arnaldo 715, São Paulo, \\ SP 01246-904, Brasil. \\ tatifurukawa@bol.com.br
}

\begin{abstract}
This study analyzed stroke mortality in Paraná State, Brazil, in 2007, according to residence and place of death, using the official Mortality Information System. For deaths in individuals 65 years and older and from 45 to 64 years of age, 17.6\% and $29.8 \%$, respectively, occurred at home. An association was observed between region of residence and less than three years of schooling $(O R=3.96)$ four to seven years of schooling $(O R=1.75)$, and race or skin color (non-white: $O R=1.39$ ). Place of death was associated with age over $65(O R=1.69)$, less than three years of schooling $(O R=1.70)$, and residence in municipalities other than the respective headquarters of the Regional Health offices $(O R=1.33)$. The results suggested inequalities in access to hospital care at the time of death from stroke, according to region of residence and socioeconomic variables. The study supports use of the Mortality Information System as a data source to back public health policies and interventions. Further studies are needed to evaluate the factors that influence quality of patient care for stroke and to verify whether the same pattern is repeated in mortality from other causes.
\end{abstract}

Stroke; Cerebrovascular Disorders; Mortality
Tatiane Sano Furukawa 1

Thais Aidar de Freitas Mathias 2 Sonia Silva Marcon 2

\section{Introdução}

As doenças cardiovasculares são as principais causas de morte na população. Compreendem as doenças cerebrovasculares, isquêmicas do coração, hipertensivas, aterosclerose, febre reumática e outros agravos do coração ${ }^{1}$. Essas doenças ocupam a primeira posição na carga de enfermidades no Brasil, contribuindo de forma expressiva para a mortalidade e morbidade precoces. São responsáveis por 85 milhões de anos de vida perdidos ajustados pela incapacidade 2 . Segundo dados do Ministério da Saúde, no Brasil em 2007, entre as doenças cardiovasculares as cerebrovasculares ocuparam o primeiro lugar na mortalidade com coeficiente de 52,61 óbitos por 100 mil habitantes 3 . A principal forma de doenças cerebrovasculares é o acidente vascular cerebral (AVC) que pode ser isquêmico ou hemorrágico, com significativa importância a partir de 40 anos de idade e grande número de internações, seqüelas e óbitos a partir de 65 anos.

Estudos sobre a mortalidade por doenças cerebrovasculares são necessários para que se conheça sua distribuição por localidade, a fim de subsidiar políticas de atenção aos agravos agudos e crônicos mais prevalentes. Além da distribuição dos óbitos por causa, sexo e idade, outras variáveis devem ser exploradas em estudos de mortalidade. A região de residência do falecido e o local de ocorrência do óbito podem ser utili- 
zados como indicadores da qualidade do atendimento e do acesso aos serviços de saúde 4 .

No início do século XX era mais comum as pessoas morrerem em suas casas. A partir dos anos 1950, em muitos países industrializados o óbito passou a ocorrer cada vez mais nos hospitais ou em outras instituições de saúde ${ }^{5}$. Mais recentemente, entretanto, nesses países, tem sido observado declínio no número de óbitos nos hospitais. Paralelamente a isso, houve um aumento de óbitos nos domicílios ou em outros estabelecimentos de saúde, como as nursing homes, instituições presentes no sistema de saúde na América do Norte. Essa queda na porcentagem de óbitos ocorridos no hospital vem acontecendo, tanto para a mortalidade por todas as causas 6 como por causas específicas, como os óbitos por AVC e infarto agudo do miocárdio 7. Entre 1989 e 2002, no Estado de Washington nos Estados Unidos, a proporção de óbitos hospitalares diminuiu de $44,2 \%$ para $35,4 \%$, enquanto que a proporção de óbitos no domicílio aumentou de $24,1 \%$ para $31,1 \%$, nos dois anos analisados 6 .

Enquanto nos países industrializados os óbitos ocorridos no hospital estão diminuindo, no Brasil a tendência é de aumento. De 1979 a 2004, os óbitos por todas as causas ocorridos no hospital aumentaram de $58,3 \%$ para $65,5 \%$, e o percentual de óbitos por doenças cerebrovasculares no hospital aumentou de $75,6 \%$ para $80,4 \%$, nos dois anos, respectivamente (Departamento de Informática do SUS. Informações em saúde 2009. http://www2.datasus.gov.br/DATASUS/, acessado em 18/Jan/2010). No Município de Londrina (Paraná), de 1936 a 1982, houve um aumento acentuado de óbitos por todas as causas ocorridos em ambiente hospitalar, principalmente a partir da década de 1970 8. Os não residentes em Londrina morriam proporcionalmente mais no hospital (96,9\%) em 1980, se comparados aos residentes (69,9\%) em $1982{ }^{8}$.

À medida que a população envelhece aumenta a utilização de serviços, principalmente as internações ${ }^{9}$ de média e alta complexidade, e a análise do perfil de mortalidade por causas pode acrescentar conhecimentos sobre as variações regionais na situação de saúde da população. Apesar de o Estado do Paraná apresentar, em geral, indicadores socioeconômicos e de saúde melhores que a média do Brasil 10, ainda não existem estudos sobre a mortalidade por causas segundo residência e local de ocorrência do óbito que podem indicar desigualdades no acesso aos serviços de saúde ${ }^{4}$. Diante disso, o objetivo deste trabalho foi analisar a mortalidade por doenças cerebrovasculares no Estado do Paraná segundo dados demográficos, local de residência e de ocorrência do óbito.

\section{Método}

Estudo ecológico, descritivo exploratório em que foram analisados todos os óbitos por doenças cerebrovasculares de residentes no Estado do Paraná, com 45 anos e mais de idade, em 2007. Os dados de mortalidade foram obtidos no Sistema de Informações sobre Mortalidade (SIM), e os populacionais nas estimativas para 2007 do Ministério da Saúde (Departamento de Informática do SUS. Informações em saúde 2008. http:// tabnet.datasus.gov.br/cgi/tabcgi.exe?ibge/cnv/ poppr.htm, acessado em 18/Jan/2010).

O Estado do Paraná está dividido em 399 municípios agrupados em 22 Regionais de Saúde, e para cada uma delas existe um município sede. Essas Regionais exercem função de apoio e cooperação técnica, além de função intermediária para investimentos e assessoria aos municípios e consórcios. As Regionais estão, por sua vez, agrupadas em seis Macrorregionais de Saúde com o objetivo de articulá-las somando esforços na solução de problemas comuns e troca de experiências (Governo do Estado do Paraná. Regionais de Saúde, 2006. Curitiba - PR. http://www. saude.pr.gov.br/RS/index.html, acessado em 25/ Abr/2009).

Foram estudados os óbitos por doenças cerebrovasculares (códigos: I60-I69, CID-10) 1, que incluem hemorragia intracraniana (I60-I62), infarto cerebral (I63), acidente vascular cerebral não especificado se hemorrágico ou isquêmico (I64), outros acidentes vasculares cerebrais (I67I68) e seqüelas de doenças cerebrovasculares (I69). A mortalidade foi analisada segundo sexo, idade, escolaridade, raça/cor, local de ocorrência do óbito, região de residência e macrorregional de residência, por meio de frequência absoluta e relativa e coeficientes de mortalidade (por 10 mil habitantes de 45 anos e mais de idade). Para o cálculo dos coeficientes considerou-se como numerador o total de óbitos por doenças cerebrovasculares de 45 anos e mais de idade, e denominador a população residente no Estado do Paraná, em 2007, na mesma faixa etária.

Para verificar a associação entre as variáveis e a ocorrência do óbito domiciliar e em municípios não sede de Regional de Saúde, utilizou-se o teste qui-quadrado nas tabelas de contingência e o odds ratio (OR) com intervalo de 95\% de confiança (IC95\%) como medida de magnitude das associações. A análise foi realizada pelo programa Epi Info versão 6.0 (Centers for Disease Control and Prevention, Atlanta, Estados Unidos).

Para o local de ocorrência do óbito considerou-se: "hospital" e "domicílio e outros", e para a região de residência: "municípios sede de Regional de Saúde" (22 municípios) e "municípios 
não sede de Regional de Saúde” (todos os 377 não sede). Para a escolaridade, a categoria de 8 e mais anos de estudo foi considerada como referência para as outras duas categorias.

A análise univariada tem natureza descritiva, não gerando interpretações de causa e efeito entre as variáveis. Ela esclarece se a distribuição dos óbitos segundo local de ocorrência e região de residência é aleatória ou se existe padrão determinado de dependência entre estes e as variáveis selecionadas.

O projeto de pesquisa não foi submetido ao Comitê de Ética por utilizar dados públicos secundários.

\section{Resultados}

Em 2007, ocorreram 6.123 óbitos por doença cerebrovascular de residentes no Estado do Paraná, dos quais 5.834 (95,3\%) foram em pessoas acima de 45 anos (Departamento de Informática do SUS. Informações em saúde 2009. http:// www2.datasus.gov.br/DATASUS/, acessado em 18/Jan/2010). Os coeficientes de mortalidade por doenças cerebrovasculares foram maiores para as Macrorregionais de Guarapuava, Londrina e Maringá com 25,8; 25,1 e 23,3 óbitos por $10 \mathrm{mil}$ habitantes, respectivamente.

Dos 5.834 óbitos, $76,9 \%$ ocorreram na faixa de 65 anos e mais de idade; $68,2 \%$ com até 3 anos de escolaridade; $86 \%$ de raça/cor branca; 53,9\% residentes em municípios não sede de Regional de Saúde; e 73\% aconteceram no hospital. Chamou atenção a baixa escolaridade, pois $68,2 \%$ tinham até 3 anos, elevando-se para $90,9 \%$ se somados os $22,7 \%$ de $4-7$ anos de estudo (Tabela 1).

Em relação ao local de ocorrência do óbito, observou-se que Londrina foi a Macrorregional onde verificou-se o maior percentual de óbitos hospitalares $(79,7 \%)$, seguida por Maringá (78,3\%) e Ponta Grossa (72,5\%). Por outro lado, na Macrorregional de Guarapuava o percentual de óbitos por doenças cerebrovasculares fora do hospital foi o mais alto, chegando a $35,3 \%$ (Tabela 1).

A Tabela 2 mostra associação significativa de residência em municípios não sede de Regional de Saúde com a variável escolaridade menor que

Óbitos por doenças cerebrovasculares, por Macrorregional de Saúde, segundo variáveis selecionadas. Paraná, Brasil, 2007.

\begin{tabular}{|c|c|c|c|c|c|c|c|c|c|c|c|c|c|c|}
\hline \multirow[t]{2}{*}{ Variáveis } & \multicolumn{2}{|c|}{ Metropolitana } & \multicolumn{2}{|c|}{ Ponta Grossa } & \multicolumn{2}{|c|}{ Guarapuava } & \multicolumn{2}{|c|}{ Foz do Iguaçu } & \multicolumn{2}{|c|}{ Maringá } & \multicolumn{2}{|c|}{ Londrina } & \multicolumn{2}{|c|}{ Total } \\
\hline & $\mathbf{n}$ & $\%$ & $\mathbf{n}$ & $\%$ & $\mathbf{n}$ & $\%$ & $\mathbf{n}$ & $\%$ & n & $\%$ & $\mathbf{n}$ & $\%$ & $\mathbf{n}$ & $\%$ \\
\hline \multicolumn{15}{|l|}{ Idade (anos) } \\
\hline $45-64$ & 407 & 24,5 & 162 & 27,5 & 78 & 21,4 & 187 & 21,2 & 257 & 23,2 & 256 & 21,0 & 1.347 & 23,1 \\
\hline 65 e mais & 1.257 & 75,5 & 427 & 72,5 & 287 & 78,6 & 699 & 78,9 & 852 & 76,8 & 965 & 79,0 & 4.487 & 76,9 \\
\hline \multicolumn{15}{|l|}{ Escolaridade (anos) } \\
\hline Até 3 & 662 & 57,2 & 376 & 71,2 & 294 & 81,9 & 651 & 76,0 & 842 & 81,4 & 673 & 56,5 & 3.498 & 68,2 \\
\hline $4-7$ & 296 & 25,6 & 124 & 23,5 & 57 & 15,9 & 174 & 20,3 & 137 & 13,3 & 373 & 31,3 & 1.161 & 22,7 \\
\hline 8 e mais & 199 & 17,2 & 28 & 5,3 & 8 & 2,2 & 32 & 3,7 & 55 & 5,3 & 146 & 12,2 & 468 & 9,1 \\
\hline Ignorado & 507 & - & 61 & - & 6 & - & 29 & - & 75 & - & 29 & - & 707 & - \\
\hline \multicolumn{15}{|l|}{ Raça/cor * } \\
\hline Branca ** & 1.412 & 90,8 & 518 & 90,6 & 329 & 90,4 & 745 & 84,3 & 860 & 78,5 & 1.032 & 84,6 & 4.896 & 86,0 \\
\hline Não branca *** & 143 & 9,2 & 54 & 9,4 & 35 & 9,6 & 139 & 15,7 & 236 & 21,5 & 188 & 15,4 & 795 & 14,0 \\
\hline lgnorado & 109 & - & 17 & - & 1 & - & 2 & - & 13 & - & 1 & - & 143 & - \\
\hline \multicolumn{15}{|c|}{ Local de ocorrência do óbito* } \\
\hline Hospital & 1.143 & 68,7 & 427 & 72,5 & 236 & 64,7 & 611 & 69,0 & 868 & 78,3 & 973 & 79,7 & 4.258 & 73,0 \\
\hline Domicílio e outros & 520 & 31,3 & 162 & 27,5 & 129 & 35,3 & 275 & 31,0 & 240 & 21,7 & 248 & 20,3 & 1.574 & 27,0 \\
\hline Ignorado & 1 & - & - & - & - & - & - & - & 1 & - & 0 & 0 & 2 & - \\
\hline \multicolumn{15}{|l|}{ Região de residência } \\
\hline Sede & 997 & 59,9 & 294 & 49,9 & 123 & 33,7 & 349 & 39,4 & 419 & 37,8 & 506 & 41,4 & 2.688 & 46,1 \\
\hline Não sede & 667 & 40,1 & 295 & 50,1 & 242 & 66,3 & 537 & 60,6 & 690 & 62,2 & 715 & 68,6 & 3.146 & 53,9 \\
\hline
\end{tabular}

* Excluídos os óbitos com variáveis ignoradas;

** Raça/cor branca: branca + amarela;

*** Raça/cor não branca: negra + parda + indígena. 
Mortalidade por doenças cerebrovasculares por região de residência e variáveis selecionadas. Paraná, Brasil, 2007.

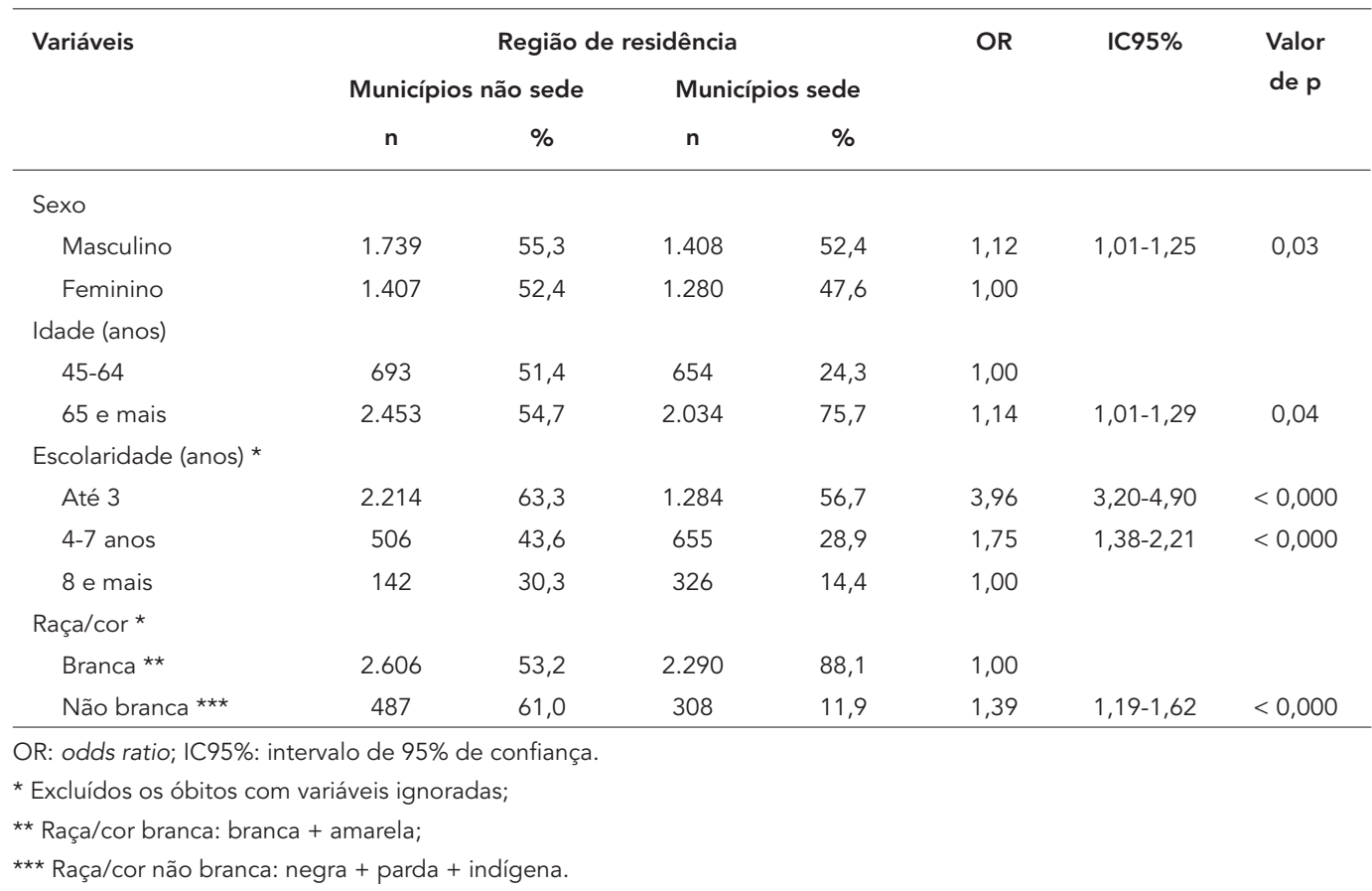

3 anos $(\mathrm{OR}=3,96)$ e de 4-7 anos $(\mathrm{OR}=1,75)$, e com a raça/cor não branca $(\mathrm{OR}=1,39)$.

Houve associação significativa da ocorrência de óbitos fora do hospital e idade acima de 65 anos $(\mathrm{OR}=1,99)$; escolaridade menor que 3 anos $(\mathrm{OR}=1,70)$; e residência em municípios não sede de Regional de Saúde (OR= 1,33) (Tabela 3).

Quanto às variáveis com declarações ignoradas, escolaridade e raça/cor foram as que apresentaram o maior percentual de ausência de informação (12,1\% e 2,4\%, respectivamente). A Macrorregional Metropolitana (Curitiba) foi a que apresentou o maior percentual de não declaração da variável escolaridade, com 30,5\%, e Guarapuava o menor, com 1,6\% (Tabela 1).

\section{Discussão}

Os resultados mostraram maior proporção de óbitos por doenças cerebrovasculares em pessoas de raça/cor branca, idade avançada, com baixa escolaridade e ocorrido no hospital. Perfil que também foi identificado em estudo realizado no mesmo ano na cidade de Maringá 11. Em relação ao alto percentual de raça/cor branca, é importante lembrar que para os estados da Região Sul do Brasil esta predominância pode ser resultado da distribuição demográfica de raças, por serem estados colonizados principalmente por população européia.

A maior taxa de mortalidade por doenças cerebrovasculares em pessoas com mais de 65 anos reflete o aumento do risco à medida que a população envelhece. Estudo realizado pelo Ministério da Saúde 3 , mostrou para todas as regiões brasileiras tendência de aumento dos coeficientes de mortalidade com a idade, e valores bem mais elevados a partir de 80 anos. No idoso é grande a prevalência de hipertensão arterial, fator que contribui para a incidência de doenças cerebrovasculares, além do etilismo e tabagismo frequentes neste grupo populacional, principalmente no sexo masculino 12 .

Quanto à baixa escolaridade, estudo sobre mortalidade por doenças cardiovasculares no Brasil também mostrou que em metade dos municípios estudados menos de $10,7 \%$ dos adultos de 25 anos e mais de idade tinham mais de 12 anos de estudo 13. Além disso, nesse mesmo estudo foi encontrada correlação inversa da mortalidade por doenças cerebrovasculares e escolaridade e renda ${ }^{13}$. As condições socioeconômicas precárias podem contribuir para a desvantagem às informações sobre saúde, para aquisição de alimentos saudáveis, menor disponibilidade de locais adequados para a prática de atividade física e para o acesso e/ou consumo de tecnolo- 
Mortalidade por doenças cerebrovasculares por local de ocorrência do óbito segundo variáveis selecionadas. Paraná, Brasil, 2007

\begin{tabular}{|c|c|c|c|c|c|c|c|}
\hline \multirow[t]{3}{*}{ Variáveis } & \multicolumn{4}{|c|}{ Local de ocorrência do óbito * } & \multirow[t]{3}{*}{ OR } & \multirow[t]{3}{*}{ IC95\% } & \multirow[t]{3}{*}{ Valor de $p$} \\
\hline & \multicolumn{2}{|c|}{ Domicílio e outros } & \multicolumn{2}{|c|}{ Hospital } & & & \\
\hline & $\mathbf{n}$ & $\%$ & $\mathrm{n}$ & $\%$ & & & \\
\hline \multicolumn{8}{|l|}{ Sexo } \\
\hline Masculino & 856 & 27,2 & 2.290 & 72,8 & 1,02 & $0,91-1,15$ & 0,70 \\
\hline Feminino & 718 & 26,7 & 1.968 & 73,3 & 1,00 & & \\
\hline \multicolumn{8}{|l|}{ Idade (anos) } \\
\hline $45-64$ & 237 & 17,6 & 1.110 & 82,4 & & & \\
\hline 65 e mais & 1.337 & 29,8 & 3.148 & 70,2 & 1,99 & $1,70-2,33$ & $<0,000$ \\
\hline \multicolumn{8}{|c|}{ Escolaridade (anos) * } \\
\hline Até 3 & 1.038 & 29,7 & 2.459 & 70,3 & 1,70 & $1,33-2,18$ & $<0,000$ \\
\hline $4-7$ & 268 & 23,1 & 893 & 76,9 & 1,21 & $0,92-1,59$ & 0,16 \\
\hline 8 e mais & 93 & 19,9 & 375 & 80,1 & 1,00 & & \\
\hline \multicolumn{8}{|l|}{ Raça/cor * } \\
\hline Branca & 1.316 & 26,9 & 3.579 & 73,1 & 1,00 & & \\
\hline Não branca & 229 & 28,8 & 566 & 71,2 & 1,10 & $0,93-1,30$ & 0,26 \\
\hline \multicolumn{8}{|c|}{ Região de residência } \\
\hline Sede & 644 & 24,0 & 2.043 & 76,0 & 1,00 & & \\
\hline Não sede & 930 & 29,6 & 2.215 & 70,4 & 1,33 & $1,18-1,50$ & $<0,000$ \\
\hline
\end{tabular}

OR: odds ratio; IC95\%: intervalo de 95\% de confiança.

* Excluídos os óbitos com variáveis ignoradas;

** Raça/cor branca: branca + amarela;

*** Raça/cor não branca: negra + parda + indígena.

gias de assistência à saúde 14 . Por outro lado, o alto percentual de baixa escolaridade pode também refletir o que ocorre na população idosa, composta em geral por pessoas pertencentes a uma geração que quando crianças e adultos não tinham acesso fácil à escola, provavelmente vivendo em área rural do Estado do Paraná ou em outros estados.

Vários estudos apontam que as condições crônicas tendem a acometer mais intensamente as camadas de menor nível socioeconômico 13 . No Brasil, foi observada associação inversa entre condições socioeconômicas (indicadas pela renda, escolaridade e moradia) e mortalidade por doenças cerebrovasculares e hipertensivas 13. Em Salvador (Bahia), cidade com elevada frequência de baixa escolaridade e renda, foi crítico o perfil de risco cardiovascular da população adulta 15 . Em Bambuí (Minas Gerais), foi encontrada associação entre escolaridade menor que 4 anos e maior risco para doenças cardiovasculares em adultos 16 . A presença simultânea de dois ou mais fatores de risco para doenças cerebrovasculares também foi verificada em pessoas com menor escolaridade 15,16 e da raça negra 15 .
As análises das desigualdades em saúde segundo raça/cor vêm sendo possíveis quando os dados de nascimento do Sistema de Informações sobre Nascidos Vivos (SINASC) e de óbito, do SIM, são utilizados. Com essas análises vem sendo evidenciados indicadores desfavoráveis para a população negra. Essa população já vive em situação de exclusão social, que a coloca em posição altamente desvantajosa, em condições de vulnerabilidade e exposta a maiores riscos devido ao acúmulo das desigualdades 17 .

Neste estudo, foi observado que o percentual de óbitos ocorridos no domicílio foi maior para os municípios não sede de Regional de Saúde. Considerando que o indivíduo deva ter atendimento médico hospitalar especializado na ocorrência ou tratamento das complicações de um agravo cerebrovascular, esses óbitos podem indicar alguma dificuldade ou desvantagem no acesso da população aos serviços de saúde.

Além disso, houve associação da ocorrência de óbitos domiciliares com idade, escolaridade e região de residência. Associação entre mortalidade no domicílio e residir em municípios menores e menos urbanizados tem sido descrita na 
literatura 18. A distância entre o domicílio e as instituições de saúde, de certa forma, consiste em fator limitante à sua utilização. Isso ocorre principalmente em instituições de média e alta complexidade, que estão concentradas em municípios maiores ou de referência nas Regionais de Saúde do Paraná.

O estudo da mortalidade por doenças cerebrovasculares segundo o local do óbito levanta algumas questões. O AVC normalmente é evento súbito e inesperado que muitas vezes ocorre sem socorro imediato, diminuindo a probabilidade de o óbito ocorrer em ambiente hospitalar ${ }^{18}$. Nesse caso, existe a necessidade de esclarecimentos à comunidade e treinamentos às equipes de saúde pré-hospitalares e em prontos-socorros, para o reconhecimento correto e precoce dos sinais e sintomas de um AVC possibilitando tratamento rápido e eficaz. Por outro lado, óbitos por doenças cerebrovasculares fora do hospital podem ser frequentes na população mais idosa, devido a algum tipo de sequela ou outras morbidades associadas, ou ainda por solicitação pessoal ou de familiares para não proceder a técnicas de reanimação 19, significando que morrer no domicílio seja opção do doente ou da família. Entretanto, estudos relatam que a escolha do local do óbito parece ser muito mais frequente entre pacientes com neoplasias, doenças com comportamento mais previsível e com sobrevida longa após o diagnóstico 18. Outras enfermidades com tratamentos mais complexos, agudos e agressivos, anteriores ao fim da vida, como o AVC, podem exigir mais hospitalizações, algumas vezes associadas com mortalidade hospitalar mais elevada 18.

Nos Estados Unidos, aproximadamente metade dos óbitos por doenças cerebrovasculares ocorre fora do hospital e estes são mais frequentes em idades avançadas e devido às sequelas 19 . O declínio dos óbitos hospitalares por doenças cerebrovasculares em países industrializados pode estar associado ao crescimento das nursing homes, instituições comuns nesses países, que oferecem supervisão e atendimento de enfermagem, com limitada atenção médica. Os pacientes são em geral de idade avançada, com afecções crônicas e que necessitam de cuidados especializados, mas não necessariamente de hospitalização.

Estudo realizado no Brasil, no período de 1983 a 2002, analisou a relação entre a ocorrência de mortes evitáveis de acordo com o modelo de atenção à saúde 20 . As doenças cerebrovasculares foram enquadradas no grupo de enfermidades evitáveis por diagnóstico e tratamento precoce. Os resultados apontaram que o processo de reorganização do sistema de saúde brasileiro, que prioriza a atenção básica, pode ter repercutido positivamente sobre a incidência dessas doenças, pois houve maior risco de morte de 1983 a 1992, período anterior à norma operacional que serviu para a implantação do SUS 20.

A lógica do SUS, de organização dos serviços de atenção à saúde, deve obedecer à regionalização. As instituições que oferecem tratamentos mais complexos e especializados estão localizadas frequentemente em municípios maiores e encontram-se preparadas para atender à população de uma determinada região geográfica, muitas vezes composta por grande número de municípios menores. O aprimoramento das relações entre municípios pólo que concentram tais serviços especializados e os municípios da área de abrangência da Regional deve ser tarefa constante das agendas oficiais, para que os serviços realmente atendam a toda população referenciada, garantindo quantidade e qualidade no atendimento.

O acesso aos serviços de saúde no Brasil é fortemente influenciado pela condição social das pessoas e pelo local onde residem 21. De 1998 a 2003, houve melhora no acesso para a população urbana, em todas as regiões brasileiras, exceto na Região Norte. As desigualdades geográficas, indicadas pela maior dificuldade ao uso dos serviços de saúde, pioraram no período, sendo melhor o acesso nas regiões mais desenvolvidas. A Região Sul, uma das mais prósperas do país, apresentou níveis de desigualdade extremamente altos, com chances de utilização de serviços 94,8\% maior para a população pertencente à classe de renda mais alta 21.

As desigualdades no acesso aos serviços de saúde podem refletir nos indicadores analisados neste estudo. A variação no percentual de óbitos ocorridos no hospital segundo Macrorregional no Estado do Paraná aponta para as desigualdades existentes, destacando a Macrorregional de Guarapuava, que também apresentou o maior coeficiente de mortalidade por doenças cerebrovasculares. Tais achados reforçam a necessidade de intervenções gerenciais e de aprimoramento do atendimento em saúde, para as doenças crônicas mais prevalentes na população, além das redes de referência e contrarreferência. Os fluxos de utilização dos serviços devem ser compreendidos 22 , a fim de melhorar a oferta e distribuição de hospitais e serviços de atendimento em saúde para acolher a população de forma regionalizada.

Destaca-se que o local de residência e o local do óbito mostram apenas uma faceta das desigualdades entre grupos da população. Essas questões de análise exemplificam a importância do uso do SIM para subsidiar ações e políticas públicas no Paraná. A avaliação dos óbitos por doenças cerebrovasculares, segundo diferentes 
variáveis, bem como a distribuição dos óbitos por região de residência e local de ocorrência, auxiliam no entendimento da complexa rede de fatores associados a esses óbitos, mesmo tendo sido estudado apenas o ano de 2007.

O diferencial no acesso ao hospital no momento do óbito observado neste estudo, pode encobrir dificuldade de acesso durante a vida dessas pessoas e de seus familiares e não apenas no momento da morte. Assim, são necessários estudos adicionais para avaliar as redes regionalizadas, os fluxos de utilização dos serviços, o número de leitos hospitalares por habitante, e a qualidade no atendimento ao paciente com AVC. Além disso, esses resultados também podem suscitar o interesse por outros estudos com investigação de óbitos por outras causas, a fim de verificar se o mesmo perfil se repete.

Este estudo foi realizado com base em dados secundários e por isto apresenta as limitações inerentes a tal tipo de dado. No caso do SIM, existe a possibilidade de sub-registro de óbito, espe- cificamente nos municípios pequenos. Entretanto, pode haver variáveis em branco e ignoradas, e erros de digitação dos dados no sistema. As variáveis escolaridade e raça/cor tiveram porcentuais de ausência de informação diferentes segundo as Regionais de Saúde. Em algumas Regionais eles foram elevados, considerando-se a boa reputação do Estado do Paraná quanto à qualidade dos dados do SUS.

Apesar disso, este estudo propicia subsídios para uma avaliação das estatísticas oficiais e disponibiliza dados para pesquisas posteriores, pois estudos sobre mortalidade por doenças cardiovasculares segundo local de óbito são escassos na literatura nacional. Ressalta-se que a qualidade dos dados nas Declarações de Óbito, documento básico para alimentação do SIM, desde os de identificação do falecido, como escolaridade, raça/cor, até o correto registro e digitação das causas do óbito, deve ser meta constante da equipe de saúde e setores de vigilância nos municípios e Regionais de Saúde no estado.

\section{Resumo}

A mortalidade por doença cerebrovascular foi analisada, no Paraná, Brasil, em 2007, segundo residência e local de ocorrência do óbito, pelo Sistema de Informações sobre Mortalidade (SIM). Dos óbitos de pessoas com 65 anos e mais e de 45 a 64, 17,6\% e 29,8\%, respectivamente, ocorreram nos domicílios. Houve associação da residência e escolaridade menor que três anos $(O R=3,96)$, de quatro a sete anos $(O R=1,75)$ e raçal cor não branca $(O R=1,39)$. O local do óbito foi associado com idade acima de 65 anos $(O R=1,69)$; escolaridade menor que três anos $(O R=1,70)$; e residência em municípios não sede de Regional de Saúde $(O R=$ 1,33). Os resultados indicaram desigualdades no acesso ao hospital no momento do óbito por doença cerebrovascular, segundo residência e variáveis indicativas de situação socioeconômica. Destacou-se o SIM como fonte de dados para subsidiar ações e políticas públicas. Estudos adicionais são necessários para avaliar os fatores que influenciam no atendimento ao paciente com doença cerebrovascular, e para verificar se o mesmo perfil se repete na mortalidade por outras causas.

Acidente Vascular Cerebral; Transtornos Cerebrovasculares; Mortalidade

\section{Colaboradores}

T. S. Furukawa participou na coleta, análise, interpretação e discussão dos dados, e elaboração do artigo. T. A. F. Mathias e S. S. Marcon colaboraram na concepção do estudo, interpretação dos dados, discussão e elaboração do artigo.

\section{Agradecimentos}

Ao Conselho Nacional de Desenvolvimento Científico e Tecnológico (CNPq) pelo financiamento. 


\section{Referências}

1. Organização Mundial da Saúde. Classificação estatística internacional de doenças e problemas relacionados à saúde, 10a revisão. v. 1. São Paulo: Edusp; 2001.

2. Murray CJ, Lopes AD. Mortality by cause for eight regions of the world: Global Burden of Disease Study. Lancet 1997; 349:1269-76.

3. Ministério da Saúde. Saúde Brasil 2006: uma análise da desigualdade em saúde. Brasília: Ministério da Saúde; 2006.

4. Carvalho EMF, Branco MAF. Perfil de mortalidade por doenças cardiovasculares na Região Metropolitana de Recife, segundo o Sistema de Informação sobre Mortalidade (SIM). Inf Epidemiol SUS 1996; 5:61-71.

5. Teno JM, Clarridge BR, Casey V, Welch LC, Wetle T, Shield R, et al. Family perspectives on end-of-life care at the last place of care. JAMA 2004; 291:8893.

6. Feudtner C, Silveira MJ, Shabbout M, Hoskins RE. Distance from home when death occurs: a population-based study of Washington State, 1989-2002. Pediatrics 2006; 117:e932-9.

7. Slobbe LCJ, Arah OA, Bruin A, Westert GP. Mortality in Dutch hospitals: trends in time, place and cause of death after admission for myocardial infarction and stroke. An observational study. BMC Health Serv Res 2008; 8:52.

8. Laprega MR, Manço ARX. Tendência histórica da invasão de óbitos em município da Região Sul do Brasil: período de 1936 a 1982. Cad Saúde Pública 1999; 15:513-9.

9. Soares NMV. Desigualdades na saúde reprodutiva das mulheres no Paraná. Rev Bras Epidemiol 2007; 10:293-309.

10. Mathias TAF, Mello Jorge MHP. Hospitalização e mortalidade em idosos: um exercício de análise comparativa. Ciênc Cuid Saúde 2005; 4:25-36.

11. Baptista EKK, Marcon SS, Souza RKT. Avaliação da cobertura assistencial das equipes de saúde da família às pessoas que faleceram por doenças cerebrovasculares em Maringá, Paraná, Brasil. Cad Saúde Pública 2008; 24:225-9.

12. Chor D, Lima CRA. Aspectos epidemiológicos das desigualdades raciais em saúde no Brasil. Cad Saúde Pública 2005; 21:1586-94.
13. Ishitani LH, Franco GC, Perpétuo IHO, França E. Desigualdade social e mortalidade precoce por doenças cardiovasculares no Brasil. Rev Saúde Pública 2006; 40:684-91.

14. Nogueira MC, Ribeiro LC, Cruz OG. Desigualdades sociais na mortalidade cardiovascular precoce em um município de médio porte no Brasil. Cad Saúde Pública 2009; 25:2321-32.

15. Lessa I, Araújo MJ, Magalhães L, Almeida Filho N, Aquino E, Costa MCR. Simultaneidade de fatores de risco cardiovascular modificáveis na população adulta de Salvador (BA), Brasil. Rev Panam Salud Pública 2004; 16:131-7.

16. Barreto SM, Passos VMA, Cardoso ARA, Lima Costa MF. Quantifying the risk of coronary artery disease in a community. The Bambuí Project. Arq Bras Cardiol 2003; 81:556-61.

17. Batista LE. Causas de óbito segundo raça/cor no Estado de São Paulo 1999. Boletim Epidemiológico Paulista 2005; 2:11-3.

18. Cohen J, Bilsen J, Hooft P, Deboosere P, van der Wal G, Deliens L. Dying at home or in an institution using death certificates to explore the factors associated with place of death. Health Policy 2006; 78:319-29.

19. Centers for Disease Control and Prevention. Place of death after stroke - United States, 1999-2002. MMWR Morb Mortal Wkly Rep 2006; 55:529-32.

20. Abreu DMX, César CC, França EB. Relação entre as causas de morte evitáveis por atenção à saúde e a implementação do Sistema Único de Saúde no Brasil. Rev Panam Salud Pública 2007; 21:282-91.

21. Travassos C, Oliveira, EXG, Viacava F. Desigualdades geográficas e sociais no acesso aos serviços de saúde no Brasil: 1998 e 2003. Ciênc Saúde Coletiva 2006; 11:975-86.

22. Roese A, Gerhardt TE. Fluxos e utilização de serviços de saúde: mobilidade dos usuários de média complexidade. Rev Gaúch Enferm 2008; 29:221-9.

Recebido em 10/Fev/2010

Versão final reapresentada em 27/Out/2010

Aprovado em 11/Nov/2010 